INRA Prod. Anim., 2005,18 (4), 255-263

\section{Facteurs de variation de la composition du lait à la Réunion}

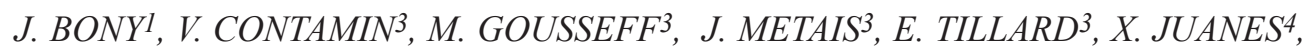 \\ V. DECRUYENAERE ${ }^{5}$, J.-B. COULON ${ }^{2}$
}

${ }^{1}$ INRA, Département PHASE, Pôle Elevage, CIRAD, Ligne Paradis, F-97410 St-Pierre-La Réunion 2 INRA, Unité de Recherches sur les Herbivores, F-63122 Saint-Genés Champanelle ${ }^{3}$ CIRAD, EMVT, Pôle Elevage, Ligne Paradis, F-97410 St-Pierre-La Réunion ${ }^{4}$ CIRAD, EMVT, Baillarguet, F-34032 Montpellier

${ }^{5}$ CRA-W, Section Systèmes Agricoles, B-6800 Libramont, Belgique Courriel : bony@cirad.fr
La production laitière à la Réunion est récente et connaît un essor très important depuis les années 1990 suite à une forte demande locale liée à la fois au changement des habitudes alimentaires, à la hausse du niveau de vie et à l'accroissement de la population.

Jusqu'au début des années 2000, l'objectif prioritaire de la filière était donc d'accroître la production laitière et pour cela, la Réunion a bénéficié de dérogations concernant l'application de la loi sur le paiement du lait à la qualité. Le développement de la production laitière s'est fait dans le cadre d'une politique d'aménagement du territoire pour fixer une population dans les zones d'altitude. De ce fait, différentes aides permettent de maintenir le prix de lait à un niveau élevé $(0,48 € / \mathrm{L})$. La mise en place progressive du paiement du lait à la qualité et les problèmes rencontrée par la filière lors de la transformation du lait ont été à l'origine de la mise en place de travaux sur les facteurs de variations de la qualité du lait dans les conditions particulières de la Réunion. Il est en effet souhaitable que le développement quantitatif de la production soit accompagné d'un développement qualitatif afin de satisfaire les exigences européennes concernant la valeur sanitaire du lait et celles des transfor- mateurs pour sa composition chimique.

Une analyse des caractéristiques chimiques (taux butyreux et protéique) et bactériologiques (numération cellulaire et germes totaux) des laits de l'ensem- ble des producteurs de l'île entre 1998 et 2001 (Bony et al 2002) a permis de mettre en évidence une très grande variabilité entre exploitations d'une part et selon la saison d'autre part (figure 1). Suite à ce premier travail, cette étude menée sur un effectif res-

Figure 1. Evolution trimestrielle des caractéristiques du lait sur l'île de la Réunion entre 1998 et 2001 (moyenne de 109 éleveurs).

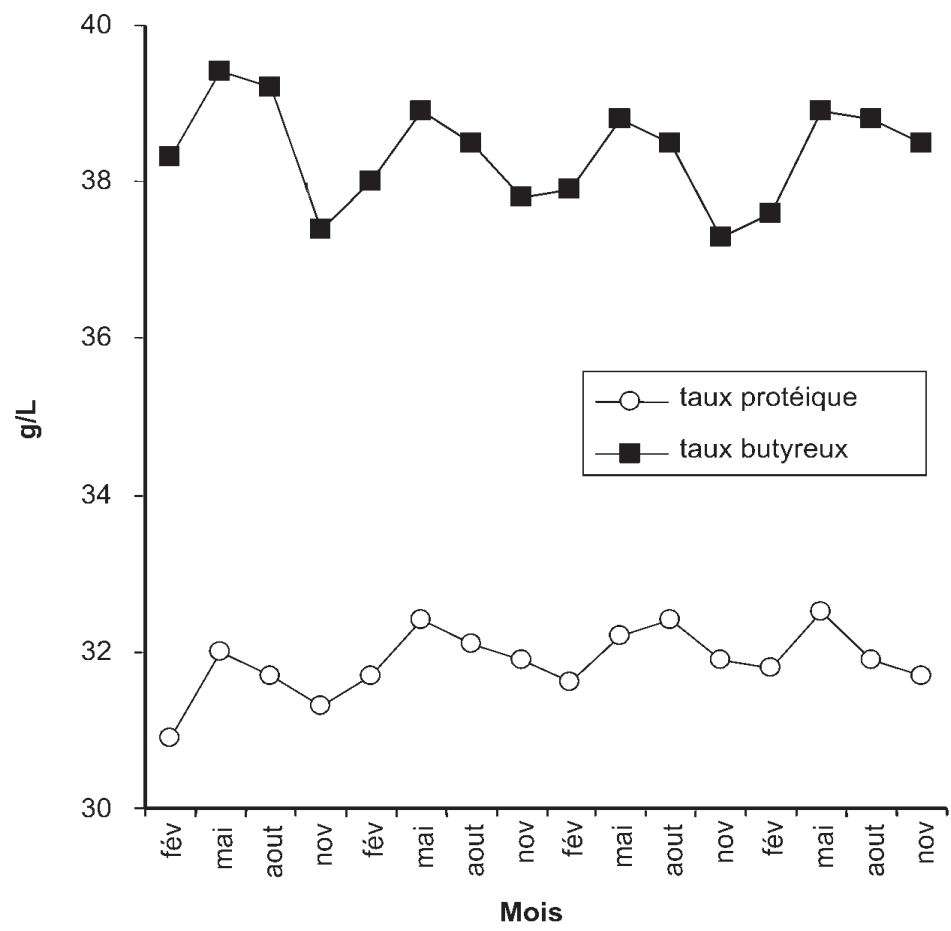


treint d'exploitations (20\% des éleveurs) a pour objectif de comprendre les principaux facteurs de cette variabilité et d'analyser leur spécificité éventuelle par rapport à ceux mis en évidence en métropole (Hoden et Coulon 1991, Coulon et al 1991, Agabriel et al 1995 et 2001).

\section{1 / Le contexte de la production laitière à la Réunion}

En 2003, la production locale a été de 22 millions de litres, soit une production encore largement inférieure à l'objectif permis par le quota global qui a été octroyé à l'île (40 millions de litres). Ce lait est produit par environ 4000 vaches réparties dans 140 élevages dont la production moyenne annuelle enregistrée par le contrôle laitier a été de $5750 \mathrm{~kg}$ en 2003. Tous ces élevages sont adhérents d'une seule coopérative qui se charge de collecter le lait et de le revendre à deux entreprises de transformation. Le lait collecté sur place représente $30 \%$ de la consommation de l'île, il sert à la production de lait de consommation, de produits frais et de fromages. Le reste des besoins en produits laitiers est satisfait par des importations de poudre de lait et de fromages.

Bien que la superficie de l'île soit réduite, les conditions pédoclimatiques, et donc les systèmes de production laitière, y sont très variables, en liaison avec l'altitude et l'exposition aux alizés. Le climat est de type tropical austral à nuances océaniques, perturbé par l'altitude et le relief. On distingue une période chaude et humide, appelée aussi saison cyclonique, de décembre à avril qui correspond à la fin du printemps, à l'été et au début de l'automne, et une période fraîche et plus sèche de mai à novembre qui correspond à la fin de l'automne, à l'hiver et au début du printemps. L'est de l'île exposé aux alizés est très arrosé ( 3 à $6 \mathrm{~m}$ d'eau /an), tandis que l'ouest, protégé par le relief central reçoit moins de $1 \mathrm{~m}$ d'eau /an. Ces différences de pluviométrie alliées aux variations d'altitude (les élevages sont répartis entre 500 et $1600 \mathrm{~m}$ ) entraînent une très grande variabilité du type de végétation et de cultures possibles $^{1}$. Dans la majorité des cas, les surfaces disponibles pour la production fourragère sont faibles, ce qui entraîne des chargements élevés ( $>4 \mathrm{UGB} / \mathrm{ha})$ et des apports d'aliments concentrés importants $(>50 \%$ de la matière sèche ingérée) dans les rations. Le rationnement individuel du concentré est peu pratiqué et souvent, quel que soit leur niveau de production ou leur stade de lactation, toutes les vaches reçoivent la même quantité de concentré. Les conditions de logement sont très variables, même si tous les élevages possèdent une aire d'alimentation abritée avec des cornadis. Pour le couchage, la majorité des élevages disposent d'aires

Tableau 1. Principales caractéristiques des exploitations enquêtées.

\begin{tabular}{|l|c|c|c|c|c|}
\hline \multicolumn{1}{|c|}{ Zone } & $\begin{array}{c}\text { Grand } \\
\text { Sud }\end{array}$ & $\begin{array}{c}\text { Hauts } \\
\text { de } \\
\text { l'Ouest }\end{array}$ & $\begin{array}{c}\text { Plaine } \\
\text { des } \\
\text { Cafres }\end{array}$ & $\begin{array}{c}\text { Plaine } \\
\text { des } \\
\text { Grègues }\end{array}$ & $\begin{array}{c}\text { Plaine des } \\
\text { Palmistes }\end{array}$ \\
\hline Nombre d'élevages & 6 & 5 & 8 & 6 & 4 \\
Pluviométrie (mm/an) & 2556 & 915 & 1702 & 2841 & 3855 \\
Température moyenne $\left({ }^{\circ} \mathrm{C}\right)$ & 19,4 & 16,1 & 13,2 & 18,7 & 16,5 \\
SAU (ha) & 11,3 & 32 & 23,3 & 8,8 & 19,8 \\
Nombre de vaches & 34 & 41 & 58 & 36 & 39 \\
Ration dominante ${ }^{1}$ & 1 & 3 & 0 & 0 & 0 \\
$\quad$ Pâturage tropical & 3 & 1 & 0 & 6 & 0 \\
$\quad$ Tropical distribué & 2 & 0 & 2 & 0 & 0 \\
$\quad$ Mixte & 0 & 1 & 6 & 0 & 4 \\
$\quad$ Tempéré distribué & & & & & \\
\hline
\end{tabular}

\footnotetext{
${ }^{1}$ Nombre d'exploitations.
}

en terre non couvertes, mais les bâtiments avec logettes commencent à se vulgariser.

Dans la majorité des exploitations, les vêlages sont étalés sur toute l'année. Les troupeaux étant de constitution récente (environ 20 ans), l'âge moyen des vaches $(5,7 \pm 0,4)$ est aussi peu variable d'un élevage à l'autre et le rang de lactation assez voisin $(3,1 \pm$ $0,5)$. Il en est de même pour la génétique qui est proche car tous les troupeaux sont constitués à partir de vaches importées de métropole qui sont réparties dans les élevages par tirage au sort. La grande majorité des troupeaux est de race Holstein $(76 \%)$ ou croisée Holstein $(18 \%)$ et il y a très peu d'élevages qui possèdent des troupeaux d'une autre race pure.

La traite est entièrement mécanisée, soit avec des pots trayeurs dans des étables entravées ou avec un quai de traite et de plus en plus dans des salles de traite avec transfert. Le lait est stocké dans des tanks réfrigérés avec une périodicité de ramassage de 2 à 4 jours.

\section{2 / Conduite de l'étude}

\section{1 / Données}

Vingt-neuf exploitations adhérentes au contrôle laitier, réparties sur l'ensemble des zones de production de l'île ont été choisies afin de constituer un échantillon représentatif des élevages locaux. Leurs caractéristiques générales sont précisées au tableau 1. Des prélèvements de lait de troupeau ont été réalisés tous les mois, entre octobre 2002 et septembre 2003, dans des tanks contenant le lait de 4 à 8 traites. Sur chaque échantillon, on a mesuré le $\mathrm{pH}$ $\left(\right.$ à $20^{\circ} \mathrm{C}$ ), les teneurs en matières grasses, en protéines, en lactose (méthode infra rouge), les teneurs en matières azotées totales et en caséines (méthode de Kjeldhal) et en urée (méthode de référence enzymatique), les teneurs en calcium (méthode par complexométrie avec de l'EDTA) et en phosphore (méthode spectrophotométrique), la lipolyse (méthode des savons de calcium), la numération cellulaire, les germes totaux et la contamination en spores butyriques. Par ailleurs, 4 fois par an (octobre, février, avril et juillet), les teneurs en sérum albumine (BSA) et en Immunoglobulines (IgG) (méthode

\footnotetext{
${ }^{1}$ Principaux fourrages cultivés à la Réunion : chloris, canne à sucre, canne fourragère dans les bas de l'île (Grand Sud et Plaine des Grègues) ; dactyle, ray-grass, brome, kikuyu dans les hauts de l'île (Hauts de l'Ouest, Plaine des Cafres, Plaine des Palmistes).
} 
d'immunodiffusion radiale) ont été déterminées.

Sur chacune des exploitations, des informations zootechniques concernant la composition du troupeau, les caractéristiques des vaches laitières et de leur conduite ont été recueillies lors de quatre passages (un par saison) ou à partir des données du contrôle laitier. $\mathrm{Au}$ cours de chacun de ces passages, des contrôles alimentaires ont été effectués, avec pesée de l'ensemble de la ration du troupeau et prélèvements d'échantillons pour mesurer les teneurs en matière sèche et les valeurs alimentaires des différents fourrages. Les analyses ont été effectuées par spectrométrie dans le proche infrarouge (SPIR) par le laboratoire de Productions Animales du CIRAD à Montpellier. La même méthode a été utilisée par le Centre de Recherche Agronomique de Libramont (Belgique) pour estimer les quantités ingérées au pâturage à partir d'une analyse d'un échantillon représentatif des fèces du troupeau (Decruyenaere et al 2002). Sur la base des prédictions SPIR, les modèles de prévisions établis d'après les équations proposées par Dulphy et al (1987) pour des fourrages tempérés, ont permis l'estimation des ingestions totales des vaches laitières et dès lors l'estimation des apports nutritionnels des rations. Les apports énergétiques ont été corrigés pour tenir compte du pourcentage d'aliments concentrés dans la ration (Vermorel et al 1987).

Les rations utilisées ont été réparties en 4 grands groupes : 1) pâturage tropical constitué essentiellement de kikuyu (Pennisetum clandestinum), 2) fourrages tropicaux distribués à l'auge, constitués essentiellement de cannes à sucre (Saccharum officinarium) ou fourragère (Pennisetum purpureum) en vert, de paille de canne et de foin de chloris (Chloris gayana), 3) ration mixte incluant à la fois des fourrages tropicaux et tempérés sans dominante particulière, 4) fourrages tempérés distribués le plus souvent sous forme d'ensilage et constitués essentiellement de dactyle, ray-grass et brome.

L'état corporel du troupeau a été noté à dire d'expert, selon trois niveaux (très bon, correct et insuffisant en se basant sur le référentiel de la note d'état).

\section{2 / Analyse des données}

L'ensemble des données a été traité à l'aide du logiciel R (R Development Core Team, 2003).

Pour mettre en relation les caractéristiques des laits et les pratiques des éleveurs, la démarche a été de constituer des classes de laits présentant des caractéristiques homogènes, puis d'analyser

Figure 2. Evolution moyenne des caractéristiques du lait au cours de l'année dans les 29 exploitations enquêtées.
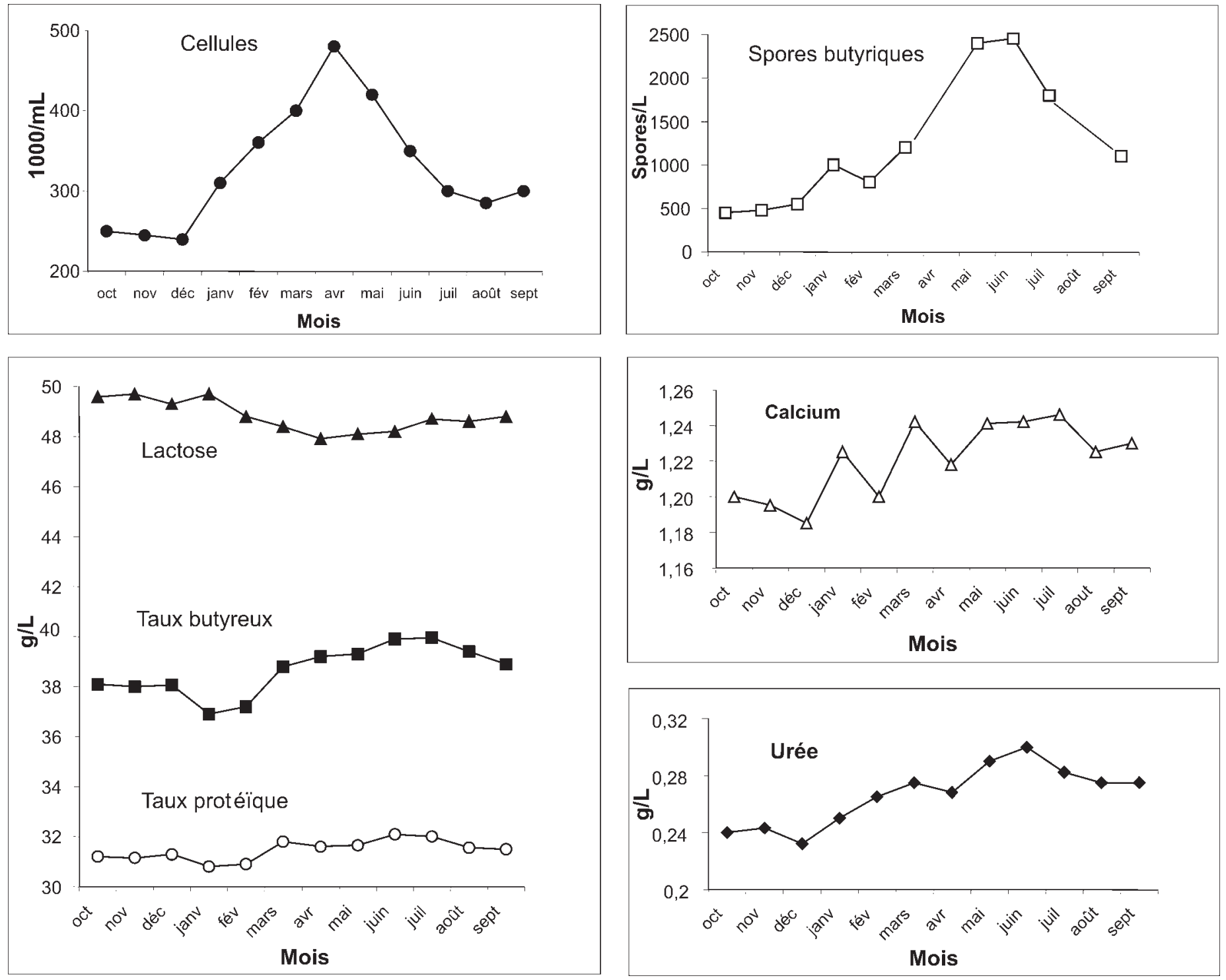
les relations entre ces classes et les caractéristiques des animaux et leur conduite. Cette analyse a été réalisée en retenant 4 périodes par exploitation, soit 116 laits au total (chaque lait correspond au lait prélevé en même temps que le passage dans l'exploitation).

Les classes de lait ont été élaborées à l'aide d'une Classification Ascendante Hiérarchique (CAH, logiciel R), construite à partir des résultats d'une Analyse en Composantes Principales $(\mathrm{ACP})$ en retenant les 6 premiers axes, qui représentaient $71,5 \%$ de la variabilité totale observée. Pour chaque lait, on a retenu 14 caractéristiques (taux butyreux, taux protéique, lactose, caséines/protéines, urée, calcium, phosphore, BSA, IgG, cellules, germes totaux, spores butyriques, lipolyse, $\mathrm{pH}$ ).

\section{3 / Résultats}

\section{1 / Caractéristiques des laits}

En moyenne, les laits analysés chez les 29 éleveurs contenaient 38,7 g/L de matières grasses, $31,5 \mathrm{~g} / \mathrm{L}$ de protéines, 324000 cellules/mL et 25300 ger$\mathrm{mes} / \mathrm{mL}$. Ces valeurs correspondent tout à fait à la moyenne de l'ensemble des exploitations de l'île. La variabilité a été très différente selon les constituants : la teneur en lactose, le rapport caséines/protéines, le $\mathrm{pH}$ et la lipolyse du lait ont présenté de faibles variations $(\mathrm{CV}<3 \%)$. A l'inverse, les teneurs en IgG, BSA et urée, ainsi que les numérations cellulaires et la teneur en spores butyriques ont été très variables. Cette variabilité dépend en partie de la saison (figure 2). C'est à la fin de la saison cyclonique et au cours de la saison fraîche (avril à septembre) que les laits sont les plus riches en matières grasses et en protéines. La saison des pluies (février à mai) est caractérisée par un pic de cellules et de germes totaux ainsi que par une diminution du rapport caséines/protéines et du taux de lactose. La concentration en spores butyriques est maximale en mai-juin.

Sur l'ensemble des échantillons analysés, la teneur en caséines a été liée de manière très étroite et linéaire à la teneur en MAT $\left(\mathrm{R}^{2}=0,91\right)$ et en protéines $\left(\mathrm{R}^{2}=0,84\right)$. Le rapport caséines/protéines a été indépendant du niveau du taux protéique du lait. Il a été plus bas $(\mathrm{P}<0,05)$ avec les numérations cellulaires les plus élevées $(83,1 \%$ pour les numérations cellulaires inférieures à 200000 contre 81,6\% pour celles supérieures à 500 000). A l'inverse, les teneurs en lactose ont été plus faibles, et celles en IgG et en BSA plus élevées $(\mathrm{P}<0,05)$, lorsque la numération cellulaire était plus forte (respectivement 49,3, 0,38 et 0,16 pour les numérations inférieures à 200000 et $48,1,0,52$ et 0,20 pour les numérations supérieures à 500 000). Le taux de calcium a varié dans le même sens que le taux protéique $\left(\mathrm{R}^{2}=0,23\right)$.

\section{2 / Typologie des laits selon leurs caractéristiques}

Le premier plan de l'ACP (figure 3) permet de mettre en évidence deux axes de variation. Le premier axe dépend fortement des variables indicatrices de la qualité hygiénique et de la qualité protéique du lait. Il oppose des laits à forte numération cellulaire, riches en protéines totales, en urée, en calcium, en BSA et IgG, à des laits présentant un rapport caséines/protéines élevé. Le second sépare les laits selon leur composition chimique (lactose, protéines et phosphore). La classification des laits issue de cette ACP a permis de définir 5 classes dont les caractéristiques sont précisées aux tableaux 2 et 3 .
Les laits de la classe 1 sont les plus riches en matières utiles, en lactose et en minéraux. Ils présentent une numération cellulaire et une contamination en spores butyriques assez élevées (respectivement 324000 cellules $/ \mathrm{mL}$ et $1259 \mathrm{spores} / \mathrm{L})$. Ils sont produits toute l'année, à partir de rations où l'ensilage est pratiquement toujours présent. Les rations apportent des quantités d'énergie et d'azote élevées (15 UFL/j et près de $1700 \mathrm{~g}$ PDI/j) qui permettent une production laitière parmi les plus élevées $(19,1 \mathrm{~kg} / \mathrm{j})$. Ces laits sont produits dans toutes les régions de l'île, mais principalement dans des zones où la pluviométrie est faible et la température fraîche et donc le plus souvent dans la Plaine des Cafres et dans les Hauts de l'Ouest.

Les laits de la classe 2 sont légèrement moins riches en matières utiles. Ils sont caractérisés par la numération cellulaire la plus élevée (631 000 cellules $/ \mathrm{mL}$ ) des teneurs élevées en $\operatorname{IgG}$ et BSA ainsi qu'en urée, un faible rapport caséines/protéines et le moins de lactose. Plus de la moitié de ces laits sont produits en automne et aucun n'est produit au printemps. Les rations correspondantes associent du pâturage et de l'ensilage d'herbe. Les apports

Figure 3. Représentation des 14 variables actives dans le premier plan de l'ACP (ce plan explique 33,3\% de l'inertie totale).

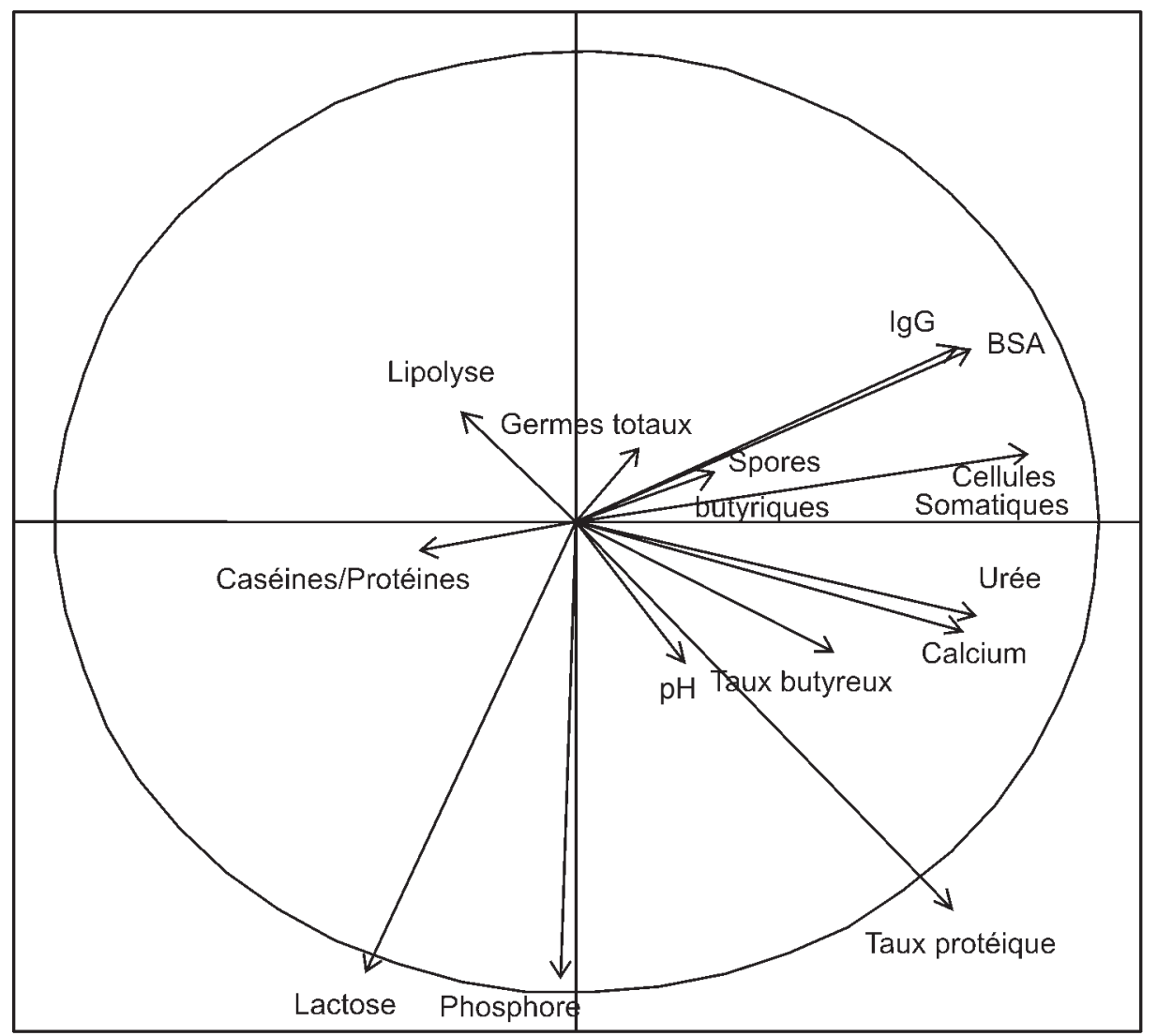


Tableau 2. Caractéristiques chimiques des classes de lait.

\begin{tabular}{|l|c|c|c|c|c|c|c|}
\hline \multicolumn{1}{|c|}{$\begin{array}{c}\text { Classes de laits } \\
\text { Effectifs }\end{array}$} & $\mathbf{1}$ & $\mathbf{2}$ & $\mathbf{3}$ & $\mathbf{4}$ & $\mathbf{5}$ & $\begin{array}{c}\text { Signif. } \\
\text { (1) }\end{array}$ & $\begin{array}{c}\text { Moyenne } \\
\text { Générale }\end{array}$ \\
\hline Taux butyreux (g/L) & $40.2 \mathrm{~b}$ & $38.8 \mathrm{ab}$ & $37.5 \mathrm{a}$ & $40.1 \mathrm{~b}$ & $37.5 \mathrm{a}$ & $* * *$ & 39 \\
Taux protéique (g/L) & $33 \mathrm{c}$ & $32,2 \mathrm{c}$ & $32,1 \mathrm{c}$ & $31,0 \mathrm{~b}$ & $30,1 \mathrm{a}$ & $* * *$ & 31,7 \\
Caséines / protéines (\%) & $84,1 \mathrm{~b}$ & $81,3 \mathrm{a}$ & $81,4 \mathrm{a}$ & $82,8 \mathrm{ab}$ & $83,1 \mathrm{~b}$ & $* * *$ & 82,7 \\
$\mathrm{IgG}(\mathrm{g} / \mathrm{L})$ & $0,45 \mathrm{a}$ & $0,64 \mathrm{~b}$ & $0,40 \mathrm{a}$ & $0,39 \mathrm{a}$ & $0,42 \mathrm{a}$ & $* * *$ & 0,45 \\
BSA (g/L) & $0,19 \mathrm{bc}$ & $0,23 \mathrm{c}$ & $0,14 \mathrm{a}$ & $0,20 \mathrm{c}$ & $0,16 \mathrm{ab}$ & $* * *$ & 0,18 \\
Lactose (g/L) & $49,4 \mathrm{~d}$ & $47,7 \mathrm{a}$ & $49,4 \mathrm{~cd}$ & $48,4 \mathrm{ab}$ & $48,1 \mathrm{bc}$ & $* * *$ & 48,8 \\
Urée (g/L) & $0,29 \mathrm{bc}$ & $0,33 \mathrm{c}$ & $0,27 \mathrm{ab}$ & $0,25 \mathrm{ab}$ & $0,23 \mathrm{a}$ & $* * *$ & 0,27 \\
Phosphore (g/L) & $0,97 \mathrm{~b}$ & $0,90 \mathrm{a}$ & $0,98 \mathrm{~b}$ & $0,89 \mathrm{a}$ & $0,91 \mathrm{a}$ & $* * *$ & 0,93 \\
Calcium (g/L) & $1,25 \mathrm{~b}$ & $1,23 \mathrm{~b}$ & $1,19 \mathrm{a}$ & $1,25 \mathrm{~b}$ & $1,19 \mathrm{a}$ & $* * *$ & 1,22 \\
pH & $6,79 \mathrm{a}$ & $6,75 \mathrm{ab}$ & $6,71 \mathrm{a}$ & $6,74 \mathrm{a}$ & $6,73 \mathrm{a}$ & $* * *$ & 6,75 \\
Lipolyse (meq/100g de MG) & $0,46 \mathrm{a}$ & $0,45 \mathrm{a}$ & $0,38 \mathrm{a}$ & $0,45 \mathrm{a}$ & $0,54 \mathrm{~b}$ & $* * *$ & 0,46 \\
Cellules (1000/mL)* & $324 \mathrm{~b}$ & $631 \mathrm{c}$ & $339 \mathrm{~b}$ & $339 \mathrm{~b}$ & $185 \mathrm{a}$ & $* * *$ & 331 \\
Germes totaux (1000/mL)* & 26 & 28 & 22 & 33 & 24 & $\mathrm{NS}$ & 27 \\
Spores butyriques (/L) * & $1259 \mathrm{~b}$ & $575 \mathrm{ab}$ & $724 \mathrm{ab}$ & $5888 \mathrm{c}$ & $339 \mathrm{a}$ & $* * *$ & 1096 \\
\hline
\end{tabular}

$(1)^{* * *}: P<0.001 ;$ NS : non significatif. Les valeurs avec des lettres différentes sont significativement différentes au seuil de $5 \%$.

* Pour les cellules, les germes et les spores butyriques, les calculs statistiques ont été faits sur les valeurs transformées en logarithme ; les résultats présentés sont des moyennes géométriques.

Tableau 3. Caractéristiques des élevages selon les classes de laits.

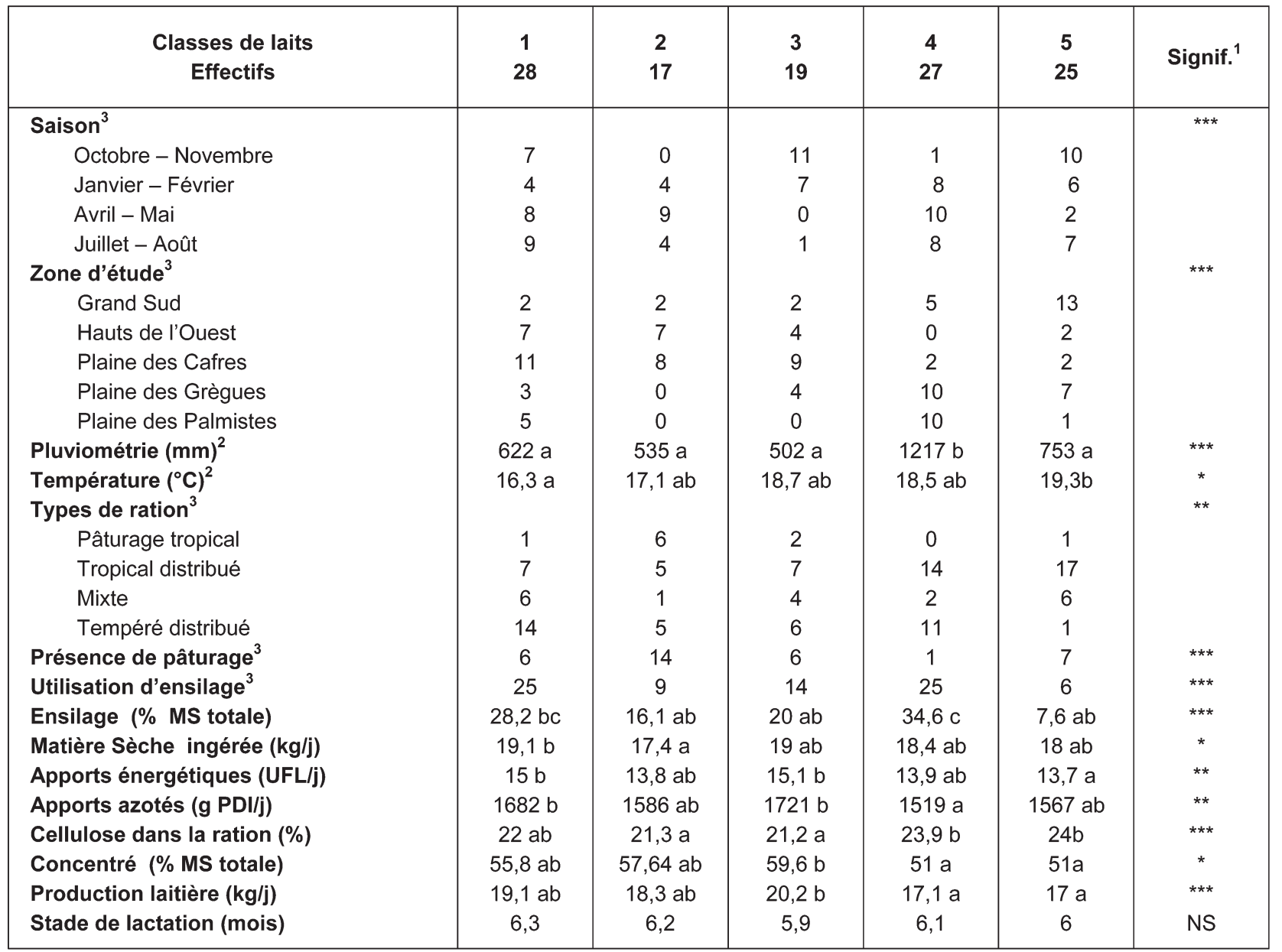

1 Signification statistique : ${ }^{* *}: \mathrm{P}<0,001 ;{ }^{* *}: \mathrm{P}<0,01 ;{ }^{*}: \mathrm{P}<0,05 ; \mathrm{NS}$ : non significatif. Les valeurs avec des lettres différentes sont significativement différentes au seuil de $5 \%$

2 La pluviométrie et la température retenues sont celles du trimestre au cours duquel le contrôle a eu lieu.

3 Nombre d'exploitations. 
énergétiques sont faibles. Ces laits sont principalement produits dans les Hauts de l'Ouest et la Plaine des Cafres, deux zones où la pluviométrie durant la période de suivi a été faible.

Les laits de la classe 3 sont caractérisés par leur faible taux butyreux et leur taux protéique, de phosphore et de lactose élevés. Ils contiennent peu de calcium, d'IgG et de BSA. Ces laits ont la particularité d'être produits d'octobre à mars, à partir de rations qui utilisent de l'ensilage dans les $3 / 4$ des cas et qui comportent la part la plus importante de concentré $(60 \%)$. Ces rations conduisent aux apports énergétiques et azotés les plus élevés, et aux productions laitières les plus fortes $(20,2 \mathrm{~kg} / \mathrm{j})$. Ces laits sont produits partout dans l'île sauf dans la Plaine des Palmistes.

Les laits de la classe 4 sont pauvres en protéines, mais riches en matières grasses et en calcium. Ils sont caractérisés par des contaminations en spores butyriques très élevées (5888 spores/L). Ils sont produits essentiellement durant les 9 premiers mois de l'année à partir de rations où l'ensilage est présent dans $93 \%$ des cas et où le pâturage est pratiquement absent. La part de concentré dans ces rations est la plus faible (51\%), les apports énergétiques et azotés sont modérés ainsi que la production laitière. Ces laits sont produits en quasi-totalité dans les zones très arrosées de l'île (Grand Sud, plaine des Grègues, plaine des Palmistes).

Les laits de la classe 5 sont les laits les plus pauvres en matières utiles et contiennent peu de minéraux et le moins d'urée. Ils présentent aussi les numérations cellulaires et les contaminations butyriques les plus faibles et le taux de lipolyse le plus élevé. Ils sont produits essentiellement d'octobre à mars, à partir de rations composées dans la grande majorité des cas de fourrages tropicaux distribués à l'auge. Comme dans la classe précédente, la part de concentré dans ces rations est la plus faible $(51 \%)$, les apports énergétiques et azotés ainsi que la production laitière sont modérés. Ces laits sont produits en grande majorité dans le Sud (Grand Sud et Plaine des Grègues) dans des zones chaudes $\left(19,3^{\circ} \mathrm{C}\right)$ et à pluviométrie moyenne (753 mm).

\section{Discussion et conclusion}

Cette étude a été réalisée dans des conditions de production du lait particulières, liées au contexte pédoclimatique, foncier et économique de l'île de la Réunion. Elle a permis de quantifier la variabilité des caractéristiques des laits de troupeaux. En moyenne, les teneurs en matières grasses, en protéines, et en lactose ont été légèrement inférieures (de 1 à $2 \mathrm{~g} / \mathrm{L}$ ) à celles observées actuellement en métropole sur des troupeaux de race Holstein de zones herbagères (CNIEL 2004, Agabriel et al 1995 et 2001). La numération cellulaire et les contaminations en spores butyriques sont supérieures aux valeurs actuellement observées dans ces mêmes zones, mais voisines de celles observées dans les années 80 (Coulon et Binet 1987, Coulon et Lilas 1988). Les autres constituants analysés (minéraux, IgG, BSA, rapport caséines/protéines, lipolyse, flore totale) présentent des valeurs voisines de celles observées actuellement en métropole (Agabriel et al 2001, Coulon et al 2002, Rémond et al 1997). Ces différentes caractéristiques ont présenté une variabilité importante, d'un troupeau à l'autre et au cours de l'année. Le stade de lactation des troupeaux qui est un facteur de variation majeur de la composition chimique du lait (Schultz et al 1990, Coulon et Remond 1991) ne permet pas ici d'expliquer cette variabilité, contrairement à ce qui est couramment observé (Agabriel et al 2001), en raison de la répartition uniforme des vêlages au cours de l'année. Cette variabilité ne peut pas être reliée non plus à la race des animaux, compte tenu de la prédominance des troupeaux Holstein sur l'île, sauf peut-être dans la classe 2, où il est probable que les taux protéiques élevés soient dus en partie à la présence dans les troupeaux de cette classe de vaches de race Brune ( 5 prélèvements sur 17). Elle s'explique donc essentiellement par la conduite des animaux, fortement liée aux conditions climatiques très marquées des différentes zones productrices de l'île.

Parmi ces facteurs, on retrouve en premier lieu les facteurs alimentaires. Ainsi, comme cela est couramment observé (Agabriel et al 1993 et 2001, Dobbelaar et al 1994) les taux protéiques élevés des classes de lait 1 et 3 peuvent être reliés aux forts apports énergétiques des rations distribuées aux vaches de ces deux classes, dont on connaît 1'effet majeur sur le taux protéique (Sutton 1989, Coulon et Rémond
1991). Ces apports sont permis par une ingestion importante d'aliments concentrés et s'accompagnent d'une production laitière élevée.

Les teneurs en spores butyriques les plus élevées (classes 1 et 4 ) se trouvent dans les laits produits avec des rations comportant une quantité significative d'ensilage d'herbe, facteur de risque important pour la contamination des laits (Coulon et Lilas 1988, Demarquilly 1998), et ce d'autant plus que les conditions climatiques (pluviométrie) et la nature des fourrages (souvent pauvres en glucides solubles) rendent plus difficile la conservation des ensilages (Paillat et al 2000) et le maintien des troupeaux en bon état de propreté : sur l'ensemble des prélèvements de laits, la contamination en spores butyriques augmente ainsi avec la proportion d'ensilage dans la ration, mais beaucoup plus fortement lorsque la pluviométrie est élevée (figure 4).

Un des facteurs de variation couramment avancé pour expliquer les variations du taux butyreux du lait est la proportion de concentré dans la ration (Journet et Chilliard 1985, Sutton 1989). Dans cette étude, la proportion de concentrés utilisés dans les rations est importante (en moyenne $55 \%$ de la matière sèche ingérée) ce qui explique le niveau des taux butyreux légèrement inférieur à celui observé actuellement en métropole en zone herbagère, en particulier dans la classe 3 où la proportion de concentré est la plus élevée. Cependant, la nature des fourrages a aussi pu modifier sensiblement le taux butyreux du lait : ainsi, l'utilisation majoritaire de fourrages tempérés dans la classe 1 s'est traduit par des taux butyreux plus élevés que pour les laits de la classe 5 où les vaches sont alimentées le plus souvent avec des fourrages tropicaux. En fait, l'examen de l'ensemble des données accompagnées d'un contrôle des rations (figure 5) montre que lorsque la ration est à base de fourrages tropicaux la proportion de concentré dans la ration n'a pas d'effet sur le taux butyreux contrairement à ce que l'on observe lorsque la ration est à base de fourrages tempérés où le taux butyreux passe en moyenne de 41,5 g/L à $37,9 \mathrm{~g} / \mathrm{L}$ lorsque la proportion de concentré passe de valeurs inférieures à $50 \%$ à des valeurs supérieures à $60 \%$. Ce résultat s'observe aussi bien lorsque les fourrages sont distribués à l'auge que lorsque les animaux pâturent. Il est possible que les caractéristiques anatomiques et chimiques particulières des fourrages tropicaux (Wilson 1994) 
Figure 4. Relations entre la contamination en spores butyriques, la proportion d'ensilage d'herbe dans la ration et la pluviométrie enregistrée au cours du trimestre du prélèvement de lait (la valeur au dessus de chaque barre représente le nombre de prélèvements).

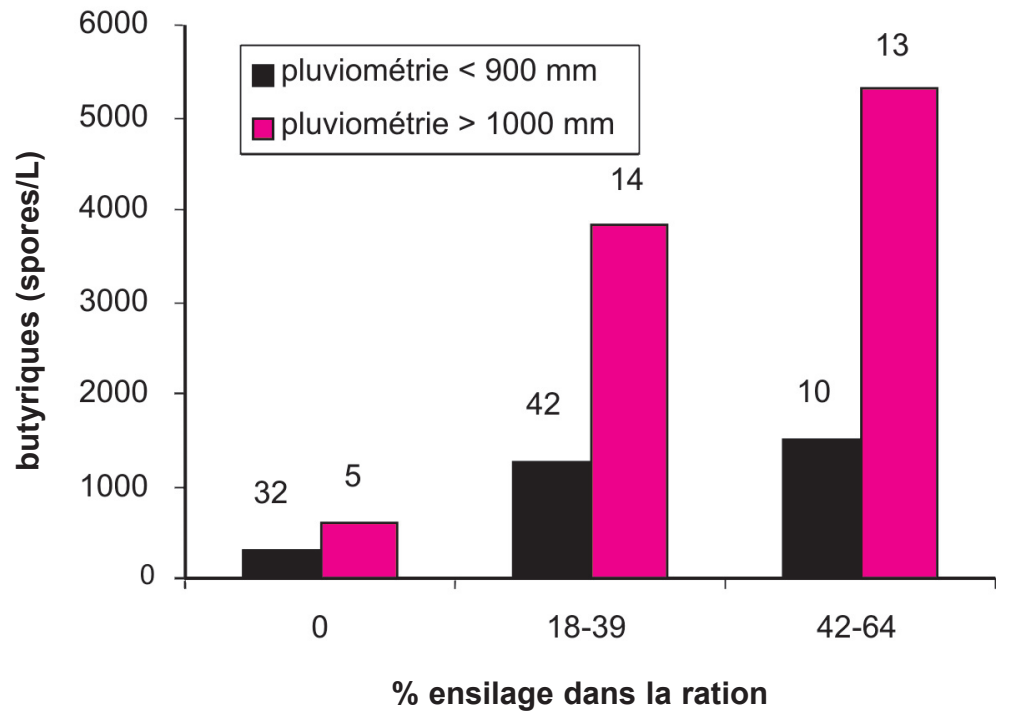

conduisent à des processus ingestifs et digestifs (augmentation de la durée de mastication et de la production de salive, augmentation du temps de séjour dans le rumen...) permettant de limiter les modifications de profils fermentaires dans le rumen habituellement observés avec des apports massifs de concentrés (Sauvant et al 1999), et en conséquence de maintenir le taux butyreux à des valeurs normales. Enfin, il est possible que les pratiques de distribution des rations mises en œuvre par certains éleveurs (mélange du concen-

tré aux fourrages, fractionnement des apports, utilisation de substances tampons...) aient contribué à éviter des chutes importantes de taux butyreux parfois observées avec des rations très riches en concentrés (Journet et Chilliard 1985, Sutton 1989, Meschy et al 2004).

Les numérations cellulaires sont élevées dans toutes les classes, excepté la classe 5. Elles sont particulièrement fortes dans la classe 2 où elles sont associées à des teneurs en lactose et à

Figure 5. Relations entre le taux butyreux et la proportion de concentrés dans la ration, en fonction de la nature des fourrages (tropicaux : $\square$, tempérés : $\square$ ).

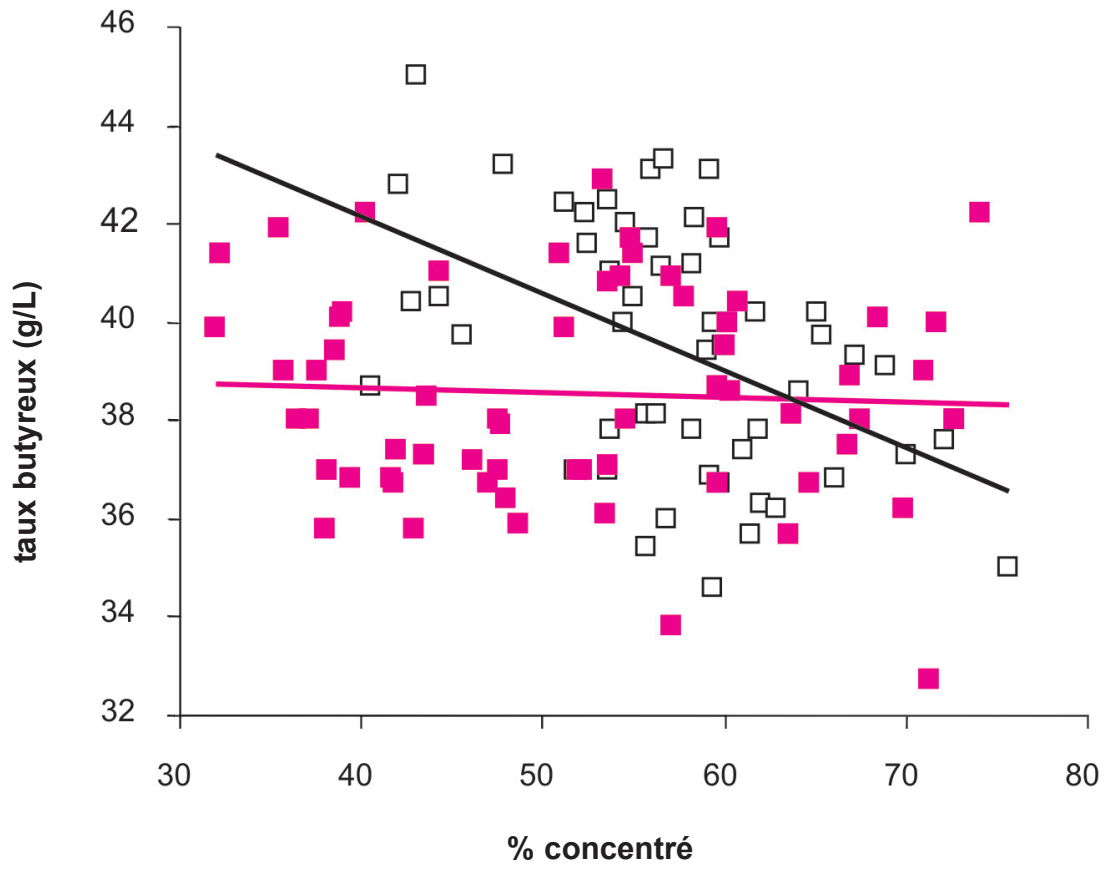

une proportion de caséines dans les protéines totales plus faibles, ainsi qu'à des teneurs en IgG et en BSA plus fortes. Ces résultats sont cohérents avec ceux de la bibliographie (Munro et al 1984, Coulon et al 2002) et illustrent les conséquences des infections mammaires sur la composition chimique du lait. Les différences entre classes s'expliquent d'une part par un effet marqué de la saison, et d'autre part essentiellement par des pratiques d'hygiène de traite et de suivi des mammites (Tillard 2001, Bony et al 2004). L'augmentation des numérations cellulaires en période estivale est fréquemment observée (Coulon et Lilas 1988, Dubeuf 1995, Agabriel et al 1995), sans que les raisons en soient clairement identifiées. Les résultats de cette étude confirment l'importance de l'effet de la saison. L'effet indirect lié à l'avancement du stade de lactation en période estivale ne peut ici être avancé. L'examen de l'évolution des numérations cellulaires au cours de l'année dans les exploitations présentant les moyennes annuelles les plus faibles et les plus élevées (figure 6), montre cependant que cet effet saison n'est pas une fatalité puisqu'il n'existe pratiquement pas chez les exploitations du groupe le plus faible en moyenne. Ce résultat suggère que les conditions estivales sont plus un révélateur de situations d'infections subcliniques préexistantes que la cause première de cette augmentation, comme cela a déjà été mis en évidence par ailleurs (Lamarche et al 2000). Il faut aussi remarquer que la numération cellulaire observée sur le lait de tank est autant une mesure de la rigueur du tri des laits mammiteux réalisé par l'éleveur que de la fréquence intrinsèque des mammites dans l'exploitation. D'une manière générale, ces pratiques de tri qui ont pu être variables d'une exploitation à l'autre, sans que nous ayons pu les quantifier, peuvent aussi expliquer les écarts observés entre exploitations.

L'examen de l'ensemble des caractéristiques du lait montre qu'il n'existe pas actuellement dans la population enquêtée de classe idéale, qui cumulerait à la fois des teneurs élevées en matières utiles (classes 1 et 2), une bonne valeur sanitaire (classe 5) et une production laitière élevée (classes 1 et 3). De même, l'examen des valeurs moyennes par exploitation et de leur évolution au cours de l'année montre clairement qu'actuellement aucun élevage ne présente tout au long de l'année des laits riches en matières utiles et pauvres en cellules et en spores buty- 
Figure 6. Evolution de la composition du lait dans les 10 exploitations présentant, pour chaque caractéristique, les valeurs moyennes annuelles les plus élevées (ㅁ) ou les plus faibles ( $\square$ ).
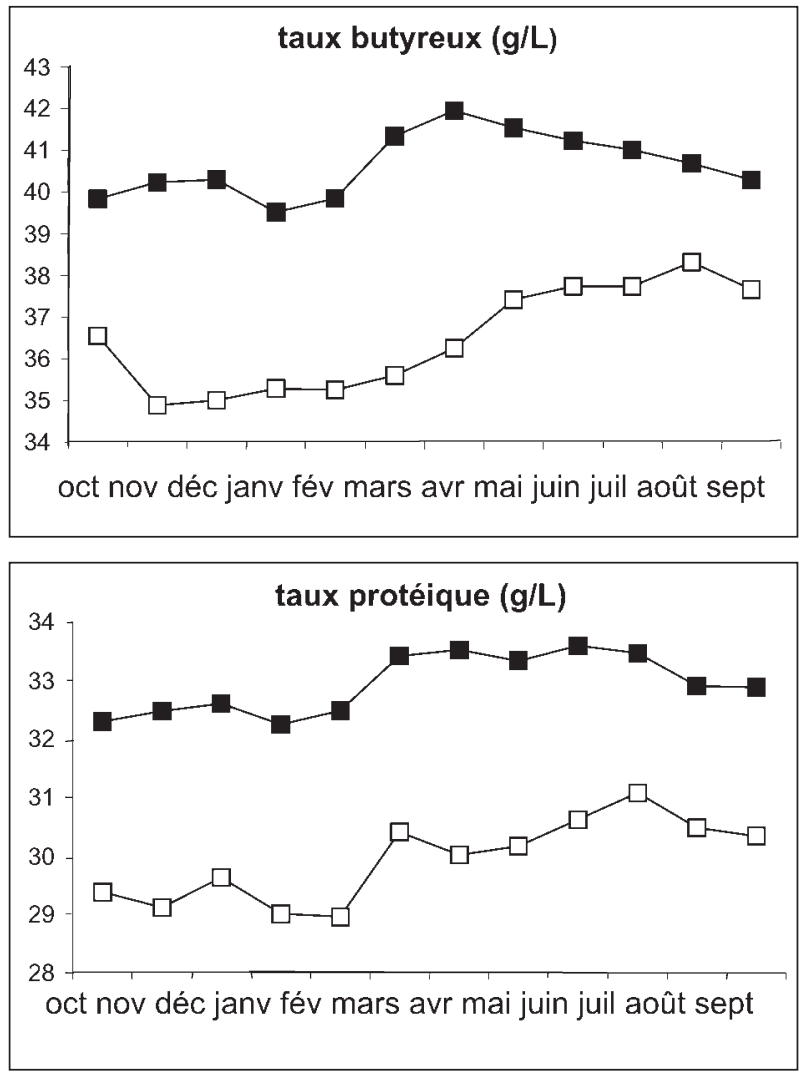
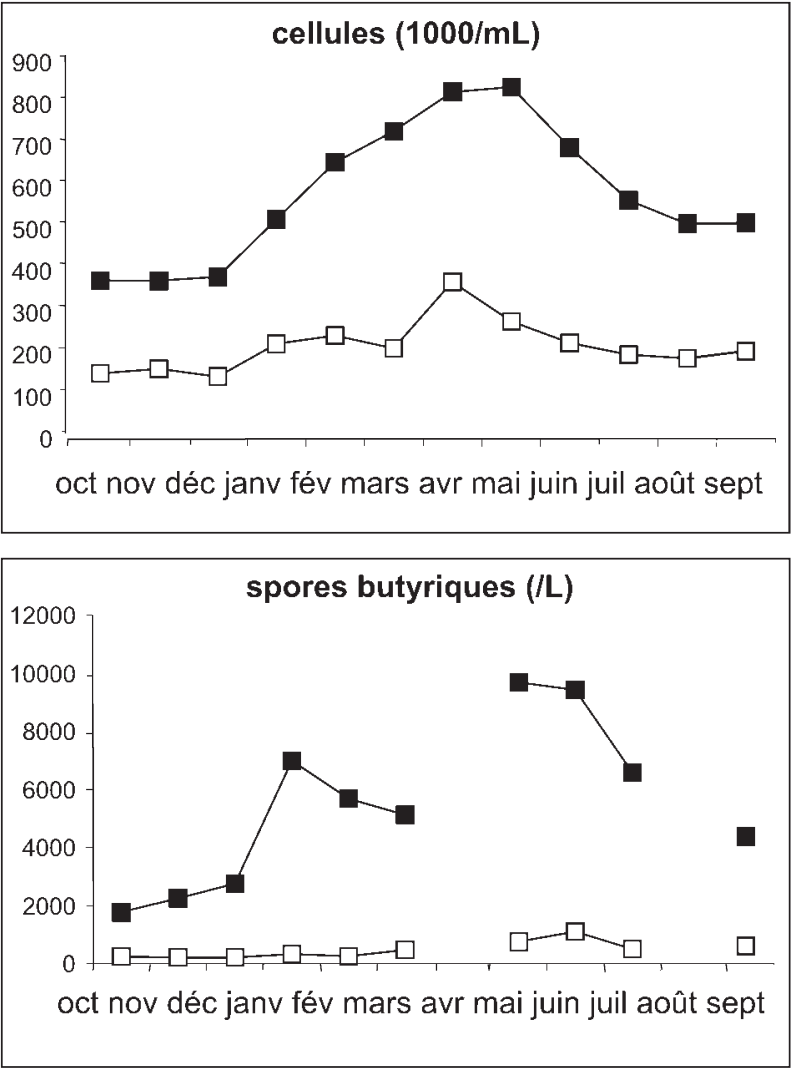

riques : ainsi, parmi les 10 exploitations présentant les taux protéiques les plus élevés (>32 g/L en moyenne sur l'année), aucune ne présentent en même temps des numérations cellulaires faibles $(<300000 / \mathrm{mL}$ en moyenne sur l'année) et des contaminations butyriques faibles $(<1000$ spores/L en moyenne sur l'année). Par contre, il montre que des exploitations sont capables d'avoir, tout au long de l'année des taux protéique ou butyreux élevés, des numérations cellulaires faibles ou des contaminations butyriques faibles (figure 6).

Il existe donc des voies d'amélioration possibles via des modifications des pratiques de récolte et de conservation des fourrages (en particulier des ensilages), de rationnement des animaux, d'hygiène du troupeau et de la traite. Ces pratiques, largement référencées en métropole, nécessitent d'être adaptées aux conditions spécifiques de l'île, et en particulier à la saison. Elles sont déjà mises en œuvre dans certaines exploitations, mais rarement de manière conjointe. Ce facteur ne doit cependant pas être considéré comme une fatalité dans la mesure où seule une classe de lait apparaît fortement liée à la saison (classe 3). Il existe donc une marge de manœuvre au sein de chaque saison, même si les pratiques à mettre en œuvre sont différentes et /ou plus ou moins difficiles selon la saison. Des travaux sont actuellement en cours pour valider la faisabilité de certaines de ces pratiques.

\section{Remerciements}

Cette étude a été réalisée avec la participation financière de la région Réunion et de l'association GALA. Nous tenons à remercier les 29 éleveurs du suivi, la Sica-Lait et l'EDE pour la mise à disposition des données et les différents laboratoires (ARIAL, CIRAD, CILAL, CRA, INRA) qui ont réalisé les analyses des fourrages et du lait.

\section{Références}

Agabriel C., Coulon J.B., Marty G., Bonaïti B, 1993. Facteurs de variation de la composition chimique du lait dans les exploitations à haut niveau de production. INRA Prod. Anim., 6, 5360.

Agabriel C., Coulon J. B., Brunschwig G., Sibra C., Nafidi C., 1995. Relations entre la qualité du lait livré et les caractéristiques des exploitations. INRA Prod. Anim., 8, 251-258.

Agabriel C., Coulon J.B., Journal C., De Rancourt B., 2001.Composition chimique du lait et systèmes de production dans les exploitations du Massif Central. INRA Prod. Anim., 14, 119128.

Bony J., Tillard E., Métais J., Angée M., 2002. Analyse des déterminants de la composition et de la qualité du lait en élevages bovins à la Réunion. Rapport d'étude préliminaire, 62p.

Bony J., Contamin V., Metais J., Nabeneza S., Tilliard E., Coulon J.B., Juanes X., 2004. Principaux facteurs qui influencent la qualité sanitaire du lait à la Réunion. Renc. Rech. Rum., $11,116$.
CNIEL, 2004. Base de données statistiques. www.cniel.com.

Coulon J.B., Binet M. 1987. Facteurs de variations du taux protéique du lait de vache en exploitation. Bull. Tech. CRZV Theix, INRA, 68, 11-18.

Coulon J.B., Lilas J.P., 1988. Composition chimique et contamination butyrique du lait : facteurs de variation dans le département de la Haute-Loire. INRA Prod. Anim., 1, 201207. 
Coulon J.B., Chilliard Y., Remond B., 1991. Effets du stade physiologique et de la saison sur la composition chimique du lait de vache et ses caractéristiques technologiques. INRA Prod. Anim., 4, 219-228.

Coulon J.B., Rémond B., 1991. Réponses de la production et de la composition du lait de vache aux variations d'apports nutritifs. INRA Prod. Anim., 4, 49-56.

Coulon J.B., Gasqui P., Barnouin J., Ollier A., Pradel P., Pomiès D., 2002. Effect of mastitis and related-germ on milk yield and composition during naturally-occurring udder infections in dairy cows. Anim. Res., 51, 383-393.

Decruyenaere V., Stilmant D., Lecomte P., Buldgen A., Dardenne P., 2002. Improvement and indirect validation of the NIRS analysis applied to faeces to measure grass intake in pasture. In: Multi-function grasslands. Quality forages, animal products and landscapes. J.L. Durand, J.C. Emile, C. Huygue and G. Lemaire (Eds). Grassland Science in Europe, 7, 196-197.

Demarquilly C., 1998. Ensilage et contamination du lait par les spores butyriques. INRA Prod. Anim., 11, 359-364.

Dobbelaar P., Scholl D.T., Roosendall E.A.M., Schukken Y.H., Brand A., 1994. Nutritionnal factors related to farm milk protein content: an observational study. Livest. Prod. Sci., 39, 253262.

Dubeuf B., 1995. Relations entre les caractéristiques des laits de troupeaux, les pratiques d'élevage et les systèmes d'exploitation dans les zones de production du Beaufort. INRA Prod. Anim., 8, 105-116.

Dulphy J.P., Faverdin P., Micol D., Bocquier F., 1987. Révision du système des unités d'encombrement. Bull. Tech. CRZV Theix, INRA, $70,35-48$

Hoden A., Coulon J. B., 1991. Maîtrise de la composition du lait : influence des facteurs nutritionnels sur la quantité et les taux de matières grasses et protéiques. INRA Prod. Anim., 4, 361-367.

Journet M., Chilliard Y., 1985. Influence de l'alimentation sur la composition du lait. 1. Taux butyreux, facteurs généraux. Bull. Tech. CRZV Theix, INRA, 60,13-23.

Lamarche A., Martin B., Hauwuy A., Coulon J.B., Poutrel B., 2000. Evolution of milk somatic cell count of cows grazing an alpine pasture according to the infection of udder by pathogens. Ann. Zootech., 49, 45-54

Meschy F., Bravo D., Sauvant D., 2004. Analyse quantitative des réponses des vaches laitières à l'apport de substances tampon. INRA Prod. Anim., 17, 11-18.

Munro G.L., Grieve P.A., Kitchen B.J., 1984. Effects of mastitis on milk yield, milk composition, processing properties and yield and quality of milk products. Aust. J. Dairy Technol., 39, 463-468.

Paillat J.M., Hassoun P., Latchimy J.Y., Brunschwig P., Lepetit J., 2000. La constitution de réserves fourragères sous forme d'ensilage. In : «L'élevage bovin à la Réunion», CIRAD (Eds), 237-244.

R Development Core Team, 2003. R: A language and environment for statistical computing, R Foundation for Statistical Computing, Vienna, Austria, ISBN 3-900051-00-3, http://www.Rproject.org.

Rémond B., Kérouanton J., Brocard V., 1997. Effets de la réduction de la période sèche ou de son omission sur les performances des vaches laitières. INRA Prod. Anim., 10, 301-315.

Sauvant D., Meschy F., Mertens D., 1999. Les composantes de l'acidose ruminale et les effets acidogènes des rations. INRA Prod. Anim., 12, 49-60

Schultz M.M., Hansen J.B., Steuernagel G.R., Kuck A.L., 1990. Variation of milk, fat, protein and somatic cells for dairy cattle. J. Dairy Sci., 73, 484-493.

Sutton J.D., 1989. Altering milk composition by feeding. J. Dairy Sci., 72, 2801-2814.

Tillard E., 2001. Etude des facteurs de variation de la qualité du lait en élevages bovins laitiers à la Réunion, Renc .Rech. Rum., 8, 94

Vermorel, M., Coulon J.B., Journet. M., 1987. Révision du système des unités fourragères. Bull. Tech. CRZV Theix, INRA, 70, 9-18.

Wilson J.R., 1994. Cell wall characteristics in relation to forage digestion by ruminants. J. Agric. Sci., 122, 173-182.

\section{Résumé}

Vingt-neuf élevages laitiers de la Réunion ont été suivis pendant 14 mois au cours des années 2002 et 2003. Des enquêtes et des contrôles d'alimentation ont été réalisés tous les trimestres pour caractériser les pratiques d'élevage et les rations distribuées. Sur des prélèvements de lait de troupeaux effectués tous les mois, des analyses de composition chimique (matières grasses, protéines, urée, lactose, minéraux,...) et de qualité hygiénique (germes totaux, cellules, spores butyriques) ont été réalisées. Au total 116 échantillons de lait correspondants à 4 passages ont été retenus pour construire une typologie des laits en 5 classes. Cette typologie a ensuite été analysée en fonction des pratiques d'élevage correspondantes. Les principaux facteurs de variation des caractéristiques des laits de troupeaux sont la saison, le niveau et la nature des apports alimentaires. Les laits les plus riches en matières utiles sont produits en hiver, essentiellement avec des rations à base de fourrages tempérés. Pour la qualité hygiénique, les meilleurs résultats sont obtenus en hiver et au printemps, dans les zones à faible pluviométrie. Aucune classe ne combine à la fois des laits riches en matières utiles, à faibles contaminations en spores butyriques et à faible numération cellulaire. Il existe donc une marge de progrès importante dans ces trois domaines, via des pratiques d'alimentation et/ou de conduite des animaux qui ont été identifiées dans cette étude.

\section{Abstract}

\section{Factors of variation of the milk composition on the Reunion Island}

In order to characterise the incidence of livestock farming practices and rations distributed to the animals on milk quality, surveys and feeding controls were carried out quarterly on twenty-nine dairy farms during 14 months (2002-2003). Herds milk samples were collected and analysed for chemical composition (fat, protein, urea, lactose, and mineral contents) and for hygienic quality (total bacteria, butyric spore and somatic cell counts). The $\mathbf{1 1 6}$ milk sample data were analysed to build a five-class milk quality typology. This typology was then confronted to the management practices. The main factors influencing the variability of herd milk were the season, and the level and nature of feeding. The milks highest in fat and proteins contents were produced in the winter, particularly when the animals were fed temperate forages. Regarding hygienic quality, the best results were obtained in the winter and spring, in areas with low rainfall. None of the five milk classes combined on a same period high fat and protein contents, and low levels of butyric spore and somatic cell counts. An important margin of progress in mastering these main quality parameters appeared. This could be realised by adapting feeding practices and/or herd management techniques that were identified in the study.

BONY J., CONTAMIN V., GOUSSEFF M., METAIS J., TILLARD E., JUANES X., DECRUYENAERE V., COULON J.-B., 2005. Facteurs de variation de la composition du lait à la Réunion. INRA Prod. Anim., 18, 255-263. 
